Ciência Florestal, Santa Maria, v. 27, n. 1, p. 47-59, jan.-mar., 2017

ISSN 1980-5098

\title{
ÍNDICE DE VALOR DE IMPORTÂNCIA DE ESPÉCIES ARBÓREAS DA FLORESTA NACIONAL DO TAPAJÓS VIA ANÁLISES DE COMPONENTES PRINCIPAIS E DE FATORES
}

\author{
IMPORTANCE VALUE INDEX OF TREE SPECIES OF TAPAJÓS NATIONAL FOREST BY \\ ANALYSIS OF PRINCIPAL COMPONENTS AND FACTORS
}

\author{
Waldenei Travassos de Queiroz ${ }^{1}$ Marcelo Lúcio Silva ${ }^{2}$ Fernando Cristovam Silva Jardim ${ }^{3}$ \\ Rodrigo Vale ${ }^{4}$ Mário Diego Rocha Valente ${ }^{5}$ João Pinheiro ${ }^{6}$
}

\begin{abstract}
RESUMO
Este trabalho avaliou, a partir das análises de componentes principais e de fatores, a aplicabilidade do modelo linear $I V I=a_{r} A_{r}+a_{2} D_{r}+a_{3} F_{r}$ para obter índices de valor de importância (IVI) para espécies arbóreas, em que $\mathrm{A}_{\mathrm{r}}$ é a abundância relativa, $D_{r}$ é a dominância relativa e $\mathrm{F}_{r}$ é a frequência relativa, comparando-os com o método univariado que considera a equação tradicional que considera $a_{1}=a_{2}=a_{3}=1$. Os dados foram analisados a partir de uma amostra de 104,04 ha, localizada na Floresta Nacional do Tapajós, município de Belterra, estado do Pará, abrangendo um conjunto de 36 conglomerados de 4 ha, formato cruz de malta, com quatro subparcelas de 1 ha. Para obter os IVIs por espécie dentro de cada conglomerado, as subparcelas foram subdivididas cada uma em 5 subsubparcelas de 0,2 ha. Os resultados mostraram que os valores obtidos pelos métodos multivariados são diferentes dos computados pela equação tradicional. Verificou-se, também, que os valores dos índices uni e multivariados variaram de forma significativa dentro da floresta, não sendo recomendável, como normalmente ocorre, inferir um valor único para a área total da floresta, pois espécies consideradas de menor importância para o total da floresta podem possuir grande importância em determinadas subáreas. $O$ uso do modelo linear com $\mathrm{a}_{1}=\mathrm{a}_{2}=\mathrm{a}_{3}=1$ não é adequado, pois, por espécie e de acordo com a sua distribuição na floresta, esses coeficientes apresentam grandes variações.
\end{abstract}

Palavras-chave: fitossociologia; inventário florestal; amostra por conglomerado.

\section{ABSTRACT}

Principal components and factor analysis were used to evaluate the applicability of the linear model $I V I=a_{t} A_{r}+a_{2} D_{r}+a_{3} F_{r}$ for obtaining indexes of value of importance $(I V I)$ for tree species, where $\mathrm{A}_{\mathrm{r}}$ is relative abundance, $\mathrm{D}_{\mathrm{r}}$ is the relative dominance and $\mathrm{F}_{\mathrm{r}}$ is the relative frequency, to compare with the standard univariate method that considers $a_{1}=a_{2}=a_{3}=1$. The data comprised a sample of 104.04 ha, located in Tapajós National Forest, Belterra municipality, state of Pará, covering of a set of 36 clusters of 4 ha each, Malta croos-shaped, with four subplots of 1 ha each. To obtain the IVI's by species within each cluster, each

1 Engenheiro Florestal, Dr., Professor Titular da Universidade Federal Rural da Amazônia, Av. Perimetral, 2501, Universitário, CEP 66077-530, Belém (PA), Brasil. waldenei.queiroz@ufra.edu.br

2 Engenheiro Florestal, MSc., Programa de Pós-Graduação em Ciências Florestais, Universidade Federal Rural da Amazônia, Av. Perimetral, 2501, Universitário, CEP 66077-530, Belém (PA), Brasil. ciencias.florestais@ufra.edu. br

3 Engenheiro Florestal, Dr., Professor Associado da Universidade Federal Rural da Amazônia, Av. Perimetral, 2501, Universitário, CEP 66077-530, Belém (PA), Brasil. fernando.jardim@ufra.edu.br

4 Engenheiro Florestal, Dr., Professor Adjunto da Universidade Federal Rural da Amazônia, Av. Perimetral, 2501, Universitário, CEP 66077-530, Belém (PA), Brasil. rodrigo.vale@ufra.edu.br

5 Estatístico, Esp., Universidade Federal do Pará, Rua Augusto Corrêa, 01, Guamá, Caixa Postal 479, CEP 66075110, Belém (PA), Brasil. mariodiego@click21.com.br

6 Estatístico, MSc., Programa de Pós-Graduação em Ciências Florestais, Universidade Federal Rural da Amazônia, Av. Perimetral, 2501, Universitário, CEP 66077-530, Belém (PA), Brasil. joaogpinheiro@yahoo.com.br

Recebido para publicação em 9/11/2012 e aceito em 16/06/2015

Ci. Fl., v. 27, n. 1, jan.-mar., 2017 
subplot was subdivided each into 5 sub-subplots of 0.2 ha each. The analysis revealed that the values obtained by multivariable differ from those obtained by the standard equation. It was also found that the values of univariate and multivariate indices vary significantly within the forest, and therefore are not recommended, as they usually are, to infer a single value for the whole forest area, because species considered of minor importance for the overall forest may be of great importance in particular subareas. The use of linear model with $a_{1}=a_{2}=a_{3}=1$ is not suitable, because of the wide variation of the coefficients among species and their spatial distribution in the forest.

Keywords: phytosociology; forest inventory; cluster sampling.

\section{INTRODUÇÃO}

A fitossociologia possibilita obter análises da estrutura florestal a partir de diversos índices existentes na literatura. O índice de valor de importância (IVI) sugerido por Curtis e McIntosh (1950), definido como a soma aritmética dos valores relativos de abundância, dominância e frequência, tem sido muito utilizado para determinar a importância ecológica das espécies, através da hierarquização em termos do grau de ocupação de sua população dentro do espaço geométrico da floresta, o que é expresso pelo número (abundância), tamanho (dominância) e distribuição espacial (frequência) dos indivíduos da população. Quanto maiores os valores de abundância, frequência e dominância mais importância terá a espécie dentro do complexo florístico da área. Cain et al. (1956) foram os pioneiros na utilização do IVI para caracterizar a estrutura de uma floresta tropical.

Lamprecht (1964) e Finol (1971) ressaltam que o conceito de estrutura florestal deve ser ampliado, devendo ser adicionado ao modelo matemático outros parâmetros como posição sociológica, regeneração natural, qualidade do fuste, etc.

Queiroz (1995) ressalta que uma espécie pode não ter importância ecológica na área florestal como um todo, mas ter uma relevância setorizada em determinados estratos. Este autor recomenda que o plano amostral contemple a possibilidade de obter o valor do IVI por unidade amostral, sugerindo para isso o uso de uma estrutura amostral por conglomerados com subdivisão das subparcelas e assim obter os índices de importância por subárea.

A construção matemática do $I V I$, baseada na soma aritmética simples dos valores de abundância, dominância e frequência, que representa uma média aritmética, foi contestada por Queiroz (1995), visto que não considera as correlações entre as variáveis envolvidas. Este autor recomenda o uso de análises de componentes principais e de fatores para definir a combinação linear que servirá como índice, resultado que corresponde a obter uma média ponderada, tal que cada variável terá seu peso de importância na construção do índice. No caso do primeiro componente principal, ou do primeiro fator, explicar significativamente a estrutura de dispersão dos dados, então os mesmos podem ser considerados como funções lineares para expressar o índice. Por outro lado, no caso de haver necessidade de usar dois ou mais componentes ou fatores, então o índice pode ser definido, se usada à análise de fatores, como o módulo da resultante da soma vetorial dos escores fatoriais ponderados pela contribuição em porcentagem dos mesmos à comunalidade total. No caso de componentes principais, ter-se-á o módulo da resultante da soma vetorial dos escores dos componentes principais ponderados pela contribuição em porcentagem dos mesmos à variância total.

Este trabalho objetivou estudar e comparar o método tradicional univariado definido como a soma aritmética dos valores relativos de abundância $\left(A_{r}\right)$, frequência $(F r)$ e dominância $\left(D_{r}\right)$ para obtenção do índice de valor de importância de espécies arbóreas na análise da vegetação da Floresta Nacional do Tapajós versus índices calculados via análises de componentes principais e de fatores.

\section{MATERIAL E MÉTODOS}

\section{Caracterização e localização da área de estudo}

A área de estudo localiza-se na Floresta Nacional do Tapajós $\left(2^{\circ} 40^{\prime}-4^{\circ} 10^{\prime} \mathrm{S} ; 54^{\circ} 45^{\prime}-55^{\circ} 00^{\prime}\right.$ W), município de Santarém no estado do Pará, Brasil. A vegetação da área é constituída, basicamente, de uma floresta alta e relativamente aberta. A variação interna do conjunto florestal abrange áreas que 
fisionomicamente se aproximam estruturalmente às matas cipoálicas que provavelmente são representativas de estágios dinâmicos (QUEIROZ, 1984). De acordo com PROJETO RADAMBRASIL (1976) na subregião dos Baixos Platôs da FLONA do Tapajós são encontradas em grande escala as espécies florestais Bertholletia excelsa Bonpl. - Lecythidaceae (castanheira-do-brasil), Bowdichia nitida Spruce ex Benth. - Fabaceae (sucupira), Ruizterania albiflora (Warm.) Marc.-Berti -Vochysiaceae (mandioqueira), Goupia glabra Aubl. - Goupiaceae (cupiúba) e Manilkara elata (Allemão ex Miq.) Monach - Sapotaceae (maçaranduba).

A área amostrada possui um tamanho de 3.745,44 ha, iniciando no km 62 da Rodovia BR-163, que liga Cuiabá a Santarém, estendendo-se no sentido norte-sul até o $\mathrm{km} 72$, distanciando-se da margem direita da estrada um mínimo de $550 \mathrm{~m}$, atingindo uma profundidade de 12,5 km em direção Leste - Oeste.

\section{Planejamento da amostragem e coleta dos dados}

A área de estudo foi dividida em 36 unidades primárias de 104,04 ha, correspondendo a um quadrado de $1.020 \mathrm{~m}$ de lado. Em cada unidade primária foi alocado um conglomerado, no qual a estrutura amostral possuía uma forma de cruz apresentando quatro subparcelas retangulares, orientadas segundo as direções Norte-Sul e Leste-Oeste (Figura 1), nas quais as subparcelas (unidades secundárias) foram tomadas com o tamanho de $500 \mathrm{~m}$ de comprimento por $20 \mathrm{~m}$ de largura, totalizando uma área de 1 ha. Cada subparcela foi subdividida em cinco subsubparcelas (unidades terciárias) de $20 \mathrm{~m}$ de largura e $100 \mathrm{~m}$ de comprimento. A área amostral total foi de 104,04 ha, que correspondeu à medição de 36 conglomerados de 4 ha cada ou 720 unidades terciárias de $20 \mathrm{~m} \times 100 \mathrm{~m}$.

Esse esquema amostral possibilitou obter o índice de valor de importância setorizado por conglomerado e para o total da área de estudo. No primeiro caso, foram consideradas como área mínima para a avaliação dos parâmetros estruturais as unidades terciárias de $0,2 \mathrm{ha}(20 \mathrm{~m} \times 100 \mathrm{~m})$, o que totalizou 20 unidades por conglomerado. No segundo caso, considerou-se área mínima o próprio conglomerado com área de 4 ha, perfazendo 36 unidades, possibilitando obter os valores dos índices para a área total.

Os dados foram coletados por Queiroz (1977) e consistiram na medição do volume comercial de todas as árvores que apresentaram DAP acima de $30 \mathrm{~cm}$. As árvores foram identificadas por parabotânico e em seguida armazenadas no herbário da EMBRAPA, Belém - PA.

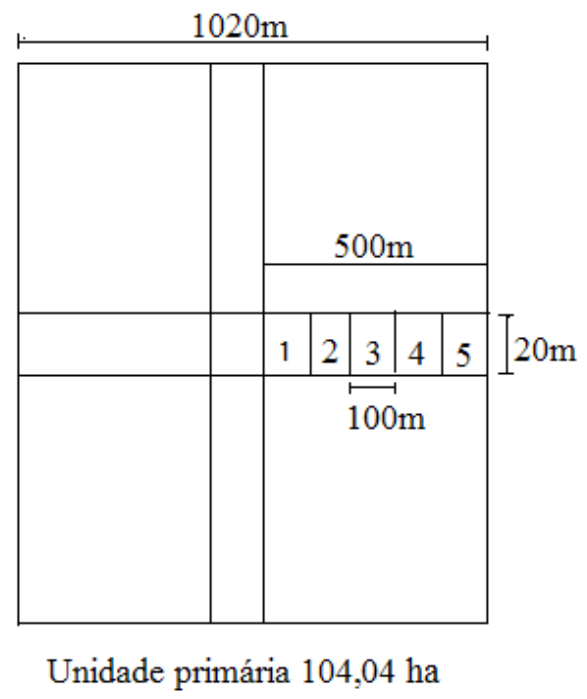

FIGURA 1: Estrutura conglomerada cruz de malta utilizada no inventário florestal da FLONA Tapajós, Município de Belterra no estado do Pará, em 1977.

FIGURE 1: Cross of Malta shaped cluster structure used in the Tapajós National Forest inventory, Belterra municipality, state of Pará, in 1977. 


\section{Índice de valor de importância (IVI) segundo Curtis e McIntosh (1950)}

Curtis e McIntosh (1950) propuseram o índice de valor de importância como a soma aritmética dos valores relativos de abundância $\left(A_{r}\right)$, frequência $(F r)$ e dominância $\left(D_{r}\right)$ para $i$-ésima espécie, ou seja, $I V I_{i}=$ $A r_{i}+D r_{i}+F r_{i}$. Esse índice, considerado absoluto, leva em consideração apenas os parâmetros da estrutura horizontal das espécies, os quais foram calculados conforme Salomão et al. (2012).

A abundância é definida como o número de indivíduos de uma espécie por unidade de área, sendo expresso em termos de relativa $(A r)$, conforme a expressão: $A_{i}=\left(n_{i} / N\right)$ x 100 , em que $n_{i}=$ número de indivíduos da $i$-ésima espécie na amostragem; $N$ é a abundância total ou número total de indivíduos por área amostral (soma das abundâncias absolutas de todas as espécies amostradas).

A frequência é um indicador do padrão de distribuição espacial dos indivíduos de uma população na floresta, sendo definida como a razão entre o número de parcelas em que ocorre pelo menos um indivíduo de uma espécie e o número total de parcelas da amostragem. $F r_{i}$ é a frequência relativa da espécie $i$ definida como a porcentagem de ocorrência da espécie em relação ao somatório das porcentagens de ocorrências considerando o número total de espécies estudadas.

A dominância é definida como o grau de ocupação que uma espécie exerce sobre o espaço volumétrico do ecossistema. Em virtude da dificuldade de avaliação desse volume, a dominância é expressa em termos de área basal. A dominância relativa $D r_{i}$ é obtida pelo quociente entre a área basal da $i$-ésima espécie, em $\mathrm{m}^{2} /$ ha, em relação à área amostrada em ha.

Para melhor explicar o comportamento da espécie serão considerados dois procedimentos, o primeiro corresponde ao IVI obtido dentro de cada um dos 36 conglomerados, enquanto que no segundo, o índice é computado acumulando os conglomerados.

\section{Índice de valor de importância pelas análises de componente principal e de fatores}

A análise de componentes principais consiste em, dado um conjunto de variáveis aleatórias, obter, por transformação linear, um número igual de novas variáveis não correlacionadas (componentes principais), tal que a soma das variâncias seja invariante. A análise de fatores estuda as relações internas de um conjunto de variáveis, substituindo-as por um número menor de variáveis hipotéticas denominadas fatores que podem ser correlacionados (fatores oblíquos) ou não correlacionados (fatores ortogonais). Esses fatores devem explicar parte significativa da variância do conjunto das variáveis originais. Valente et al. (2011) utilizaram a análise de fatores para predizer o volume de madeira para ajuste de regressão.

No caso dos primeiros componentes principais ou dos fatores explicarem parte significativa da variância total, pode-se, então, avaliá-los para cada unidade de amostra e considerá-los como os valores do índice de valor de importância.

O IVI obtido pelas análises de componente principal e de fatores foi calculado somente em relação aos valores por conglomerado. Nesse caso, os dados foram arranjados em uma matriz de dados $\mathrm{X}_{\mathrm{nxp}}$, por espécie, em que $n=36$ conglomerados e $p$ são as três variáveis respostas $A r, F r$ e $D r$. Os componentes principais das observações da matriz $\mathrm{X}_{\mathrm{n} \mathrm{x} \mathrm{p}}$ são funções lineares $I V I_{i}=a_{1 i} A r+a_{2 i} D r+a_{3 i} F r$ com variância igual a $V\left(I V I_{i}\right)=a_{v i}^{\prime} S a_{v i}$, tal que $a_{\dot{v}}^{\prime}=\left[a_{1 i}, a_{2 i}, a_{3 i}\right]$, sendo que $S=\left[s_{\dot{j}}\right]$ é a matriz amostral de covariâncias.

Para obter o primeiro componente principal calcula-se $a_{v 1}$, tal que $V\left(I V I_{1}\right)=a_{v 1}^{\prime} S a_{v 1}$, dada a condição $a_{v 1}^{\prime} a_{v 1}=1$, atinja o valor máximo.

Se o primeiro componente principal $\left(C P_{l}\right) I V I_{1}=a_{11} A b_{r}+a_{21} F_{r}+a_{31} D_{r}$ explicar parte significativa da variância total, ou seja, mais de $80 \%$, de acordo com Hair Junior et al. (2005), então o mesmo deverá ser considerado como um bom índice de valor de importância.

O segundo componente principal $\left(C P_{2}\right)$ é obtido calculando-se $a_{v 2}$, tal que $V\left(I V I_{2}\right)=a_{v 2}^{\prime} S a_{v 2}$ atinja o valor máximo atendendo às condições $a_{v 2}^{\prime} a_{v 2}=1$ e $a_{v 1}^{\prime} a_{v 2}=0$, enquanto o terceiro componente principal $\left(C P_{3}\right)$ consiste na obtenção de $a_{v 3}$ tal que $V\left(I V I_{3}\right)=a_{v 3}^{\prime} S a_{v 3}$ atinja o valor máximo atendendo às condições $a_{v 3}^{\prime} a_{v 3}=1, a_{v 1}^{\prime} a_{v 3}=0$ e $a_{v 2}^{\prime} a_{v 3}=0$.

Por outro lado, no caso de haver necessidade de usar dois ou mais componentes, então o índice 
pode ser definido como o módulo da resultante da soma vetorial dos escores dos componentes principais ponderados pela contribuição em porcentagem $\left(P_{i}\right)$ dos mesmos à variância total $\left(C P_{s v}\right)$.

$$
C P S v=\sqrt{P_{1} C P_{1}^{2}+P_{2} C P_{2}^{2}+P_{3} C P_{3}^{2}}
$$

Seja o modelo m-dimensional linear $x_{i}=\sum_{j=1}^{m} a_{i j} F_{j}+e_{i} \quad(i=1, \cdots, p)$, escrito na forma matricial:

$x_{v}=A f_{v}+e_{v}$, sendo $x_{v}^{\prime}=\left[x_{1}, x_{2}, x_{3}\right]=[A r, F r, D r]$ o vetor correspondente a $p=3$ variáveis respostas.

$A$ é a matriz de ordem $3 \times m$ dos pesos correspondentes às três variáveis respostas, sendo $m$ o número de fatores do modelo, tal que $m \leq p$.

$f_{v}^{\prime}=\left[F_{1}, F_{2}, \ldots, F_{m}\right]$ é o vetor correspondente aos fatores comuns (variáveis latentes) aleatórios, independentes e de variâncias iguais a um; $e_{v}^{\prime}=\left[e_{1}, e_{2}, \cdots, e_{p}\right]$ é o vetor inerente aos resíduos específicos aleatórios de média zero.

Seja $\Sigma=E\left(x_{v} x_{v}^{\prime}\right)=\left[\sigma_{j}\right]$ a matriz populacional de covariâncias de $A r$, Dr e Fr. Considerando pressuposições: $E\left(f_{v} e_{v}^{\prime}\right)=\phi ; E\left(f_{v} f_{v}^{\prime}\right)={ }_{m} I_{m} ; E\left(e_{v} e_{v}^{\prime}\right)=\operatorname{diag}\left(\psi_{1}, \psi_{2}, \cdots, \psi_{p}\right)$ Resulta:

$\Sigma=A A^{\prime}+\psi$ que é a matriz de covariâncias das varáveis $A r$, Dr e Fr sob o modelo fatorial.

Os elementos da matriz $A A^{\prime}$ são denominados de comunalidades das variáveis respostas $A r, D r$ e $F r$, ou seja, é a porção da variância que a variável contribui para o fator.

No uso da análise de fatores, após verificar a normalidade dos dados, é importante identificar na matriz de correlações, o número de correlações que são estatisticamente diferentes de zero. Hair Junior et al. (2005) citam que para aplicar a análise de fatores o número de correlações com valores menores que 0,30 não deve ser grande.

Mingoti (2005) cita que alguns autores recomendam que para obter um ajustamento adequado do modelo de análise de fatores é importante que a matriz de correlações inversa seja próxima da matriz diagonal. Com base nessa condição, pode-se usar o coeficiente de KMO (Kaiser-Meyer-Olkin) que mede o grau de correlação parcial entre as variáveis. De acordo com Maroco (2007), a estatística de KMO (1.1) compara as correlações simples com as correlações parciais observadas entre as variáveis, sendo dada pela fórmula abaixo, para $i \neq j$, tal que $r_{i j}$ é o coeficiente de correlação entre as variáveis $i$ e $j$ e $a_{i j}$ é o coeficiente de correlação parcial, em que os $a_{i j}$ deverão assumir valores próximos de zero, uma vez que se pressupõe que os fatores são ortogonais entre si.

$$
K M O=\frac{\sum_{i=1}^{p} \sum_{j=1}^{p} r_{i j}^{2}}{\sum_{i=1}^{p} \sum_{j=1}^{p} r_{i j}^{2}+\sum_{i=1}^{p} \sum_{j=1}^{p} a_{i j}^{2}}
$$

Os valores desse coeficiente variam de 0 a 1 e, segundo Fávero et al. (2009), quanto mais próximo da unidade mais recomendável é a aplicação da análise de fatores, pois quando as correlações parciais são próximas de zero, o coeficiente KMO tende a 1 , significando que a matriz de correlações inversa tende à diagonal. Conforme estes autores os valores abaixo de 0,50 indicam não ser recomendável à aplicação da análise de fatores.

Além do uso do coeficiente de $\mathrm{KMO}$, é recomendável também aplicar o teste de esfericidade de Bartlett para testar a hipótese nula de que as variáveis não sejam correlacionadas, ou seja, que a matriz de correlação entre as variáveis $A r, F r$ e $D r$ é uma identidade, pois se essa hipótese não for rejeitada, então não tem sentido usar a técnica de análise de fatores, pois as variáveis originais são consideradas independentes. A expressão (1.2) indicada por Bartlett (1954) com $1 \frac{1}{2} \times p(p+1)$ graus de liberdade foi usada para testar a hipótese $H_{0}: \Sigma=\Sigma_{0}$.

$$
L=\left\{1-\frac{1}{6(n-1)}[2 p+1-2 /(p+1)]\right\}\left(\operatorname{Ln}\left|\Sigma_{0}\right|-\operatorname{Ln}|S|+\operatorname{tr} S \sum_{0}^{-1}-p\right.
$$

Segundo Fávero et al. (2009), calcula-se para cada variável, similarmente à estatística KMO, uma 
medida de adequação da amostra (Measure of Sampling Adequacy - MSA). Para decidir sobre a necessidade de eliminação de variáveis no modelo de análise de fatores, pode-se obter a matriz Anti-Imagem $\mathrm{O}$ output do software SPSS apresenta os valores de MSA na diagonal principal dessa matriz. Hair Junior et al. (2005) definem a matriz de correlações anti-imagem como a matriz das correlações parciais entre as variáveis após a análise de fatores. A diagonal contém as medidas de adequação da amostra para cada variável e os demais valores são correlações parciais entre as variáveis.

A consistência interna ou confiabilidade entre as variáveis considerando as escalas dos dados pode ser avaliada pelo coeficiente de confiabilidade Alfa de Cronbach, citado em Cronbach (1951), dado pela expressão 1.3, tal que, $p$ é o número de variáveis, Cov é a média das covariâncias e $V$ é a média das variâncias.

$$
\alpha=\frac{p\left(\frac{C o v}{V}\right)}{1+(p-1)\left(\frac{C O V}{V}\right)}
$$

$\mathrm{O}$ valor $\alpha$ pode variar de 0 a 1 , significando que o valor 1 demonstra presença de consistência interna de $100 \%$ e zero significa total ausência. A literatura recomenda como limite de aceitação valores acima de 0,70 (CORRAR; PAULO; DIAS FILHO, 2007).

Neste trabalho será aplicado o método de análise de fatores denominado de máxima verossimilhança. Lawley (1940) aborda originalmente o problema da estimação na análise de fatores, tornando-a um método estatístico propriamente dito. Este autor emprega o método da máxima verossimilhança para estimar os parâmetros do modelo fatorial $x_{v}=A_{1}{ }_{v}+e_{v}$, mostrando que as estimativas dos pesos fatoriais $\hat{A}$ e as estimativas das variâncias específicas $\hat{\psi}$, tal que $E\left(e_{v}^{\prime} e_{v}\right)$, são soluções das seguintes equações matriciais implícitas:

$$
\begin{gathered}
\hat{\psi}=\operatorname{diag}\left(S-\hat{A}^{\prime} \hat{A}\right) \\
S \hat{\psi}^{-1} \hat{A}=\hat{A}\left(I+\hat{A}^{\prime} \hat{\psi}^{-1} \hat{A}\right)
\end{gathered}
$$

A principal vantagem do Método de Máxima Verossimilhança é verificar se um determinado número de fatores $m$, considerando uma amostra de tamanho $n$, está adequado para reproduzir o modelo. Segundo Bartlett (1954), $H_{0}$ é verdadeira quando a variável $U_{m}$, dada em 1.6, tem aproximadamente uma distribuição qui-quadrado com $v=1 / 2\left[\left(p \cdot U_{m}=\left(n-1-\frac{2 \dot{p}+\dddot{5}}{6}-\frac{\dot{2}}{3} m\right)\left(\operatorname{Ln}\left|\hat{A} \hat{A}^{\prime}-\hat{\psi}\right|-\operatorname{Ln}|S|\right)\right.\right.$

Os escores fatoriais não são estimados no sentido estatístico usual, pois não são parâmetros do modelo de fatores, mas valores atribuídos às variáveis hipotéticas. Atribuindo o princípio de quadrados mínimos para predizer os escores dos fatores, Thonsom (1951) estabeleceu o método para obter $m$ regressões lineares dos fatores sobre as variáveis respostas, resultando a fórmula para obter os escores fatoriais $F=X \psi^{-1} \hat{A}\left(I_{m}+\hat{A}^{\prime} \psi^{-1} \hat{A}\right)^{-1}$ que é uma matriz de ordem $N \times n$.

No caso do primeiro fator $\left(F_{I}\right)$ explicar significativamente a estrutura de dispersão dos dados, então o mesmo pode ser considerado como função linear para expressar o índice. Por outro lado, no caso de haver necessidade de usar dois ou mais fatores, então o índice pode ser definido como o módulo da resultante da soma vetorial dos escores fatoriais ponderados pela contribuição em porcentagem dos mesmos à comunalidade total $\left(F_{s v}\right)$.

$$
F s v=\sqrt{P_{1} F_{1}^{2}+P_{2} F_{2}^{2}+P_{3} F_{3}^{2}}
$$

\section{RESULTADOS E DISCUSSÃO}

Análise segundo Curtis e McIntosh (1950)

Ci. Fl., v. 27, n. 1, jan.-mar., 2017 
A espécie Lecythis lúrida (Miers) S. A. Mori foi uma das mais importantes na estrutura da floresta estudada, pois ocorreu em todos os 36 conglomerados. Observando-se a Figura 2, verifica-se que a espécie Lecythis lúrida (Miers) S.A. Mori apresentou valores de $I V I$ de 34,49, 36,07 e 42,16, respectivamente, para os conglomerados $5^{\circ}, 20^{\circ}$ e $30^{\circ}$, enquanto para as áreas acumuladas de 20 ha ( 5 conglomerados), 80 ha (20 conglomerados) e 120 ha (30 conglomerados) os valores de $I V I$ foram de 23,45, 21,64 e21,6, respectivamente. Estes resultados denotam uma grande variação no valor $I V I$ quando avaliado por conglomerado, o que é uma evidência da existência de grande variação na ocorrência da espécie na área, ou seja, essa espécie não possui uma distribuição uniforme na floresta. Se o objetivo da amostragem é identificar e caracterizar essas comunidades, então o IVI por conglomerados é o que deve ser usado. Todavia, se o objetivo da amostragem é obter uma estimativa do $I V I$ das espécies em relação à área total, o $I V I$ acumulado deve ser considerado, pois ele indicará a importância da espécie em toda a área.

A espécie Cordia bicolor A.DC. foi a espécie que apresentou a menor importância em termos dos valores de IVI na área total, inclusive com vários conglomerados apresentando IVI iguais a zero (Figura 3).

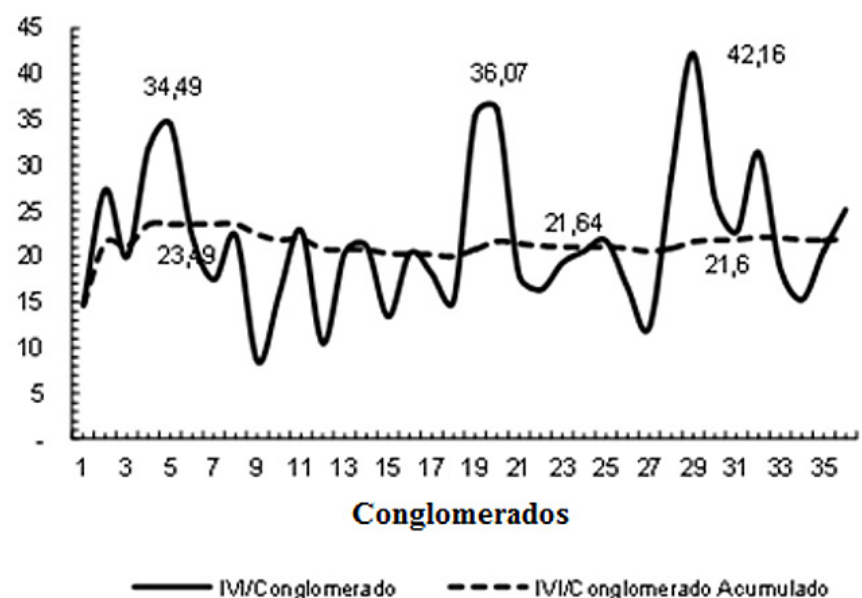

FIGURA 2: Distribuição do IVI absoluto para a espécie Lecythis lurida considerando os conglomerados isoladamente e na forma acumulada na área inventariada FLONA Tapajós, Município de Belterra PA, em 1977.

FIGURE 2: Distribution of absolute IVI for Lecythis lurida considering clusters individually and cumulatively in Tapajós National Forest inventory, Belterra municipality, in 1977.

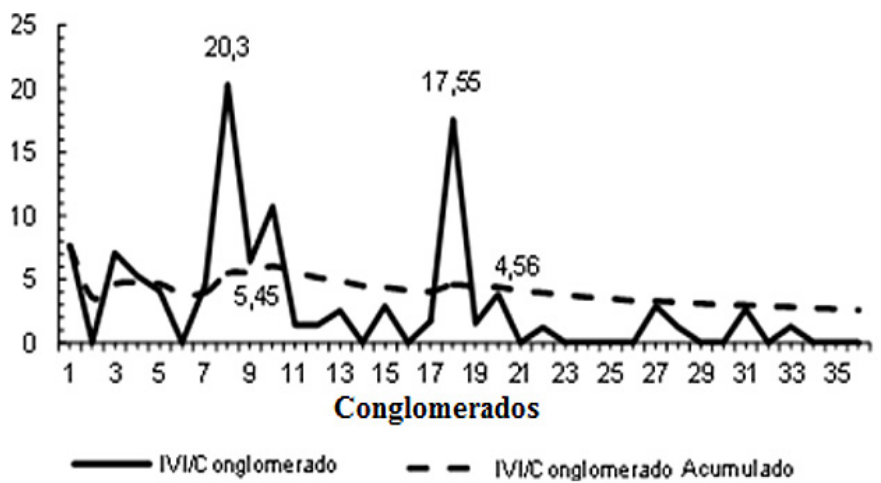

FIGURA 3: Distribuição do IVI absoluto para a espécie Cordia bicolor considerando os conglomerados isoladamente e na forma acumulada na área inventariada da FLONA Tapajós, Município de Belterra - PA, em 1977.

FIGURE 3: Distribution of absolute IVI for Cordia bicolor considering clusters individually and cumulatively in Tapajós National Forest inventory, Belterra municipality-PA state, in 1977. 
A grande variação nos valores do IVI dentro dos conglomerados foi verificada para todas as espécies encontradas na área de estudo, principalmente para as espécies pouco abundantes como C. bicolor. Os resultados apresentados mostram que espécies consideradas pouco importantes na estrutura de uma área florestal podem apresentar grande relevância quando analisadas dentro de determinadas subáreas. Esse fato deveria ser levado em consideração no planejamento da produção no manejo florestal (PEREIRA JUNIOR, 2004).

\section{Índices segundo as análises de componentes principais e de fatores}

Os resultados da análise para o primeiro componente principal, considerando as dez espécies que apresentaram os maiores valores, segundo a expressão $I V I_{i}=A r_{i}+D r_{i}+F r_{i}$, assim como os autovetores e suas porcentagens de explicação (Tabela 1) demonstram que o uso da função linear $I V I_{i}=A r_{i}+D r_{i}+F r_{i}$ ou escrita na forma $I V I_{i}=0,577350 A r_{i}+0,577350 D r_{i}+0,577350 F r_{i}$, segundo a condição $a_{1 i}^{2}+a_{2 i}^{2}+a_{3 i}^{2}=1$ , ou seja, norma do vetor dos coeficientes igual a unidade, não é recomendável, visto que os coeficientes do componente principal mostram valores diferentes entre si, resultantes das inter-relações entre variáveis aleatórias $\mathrm{Ar}, \mathrm{Dr}$ e $\mathrm{Fr}$.

TABELA 1: Resultados da análise do primeiro componente principal para as 10 espécies de maior IVI na FLONA Tapajós, Município de Belterra - PA, em 1977.

TABLE 1: Results of the analysis of the first main component for the 10 species of highest IVI in Tapajós National Forest, Belterra, PA state, in 1977.

\begin{tabular}{lccccc}
\hline \multirow{2}{*}{ Espécies } & \multirow{2}{*}{ Autovalor } & \multirow{2}{*}{$\%$} & \multicolumn{3}{c}{ Coeficientes dos autovetores } \\
\cline { 5 - 7 } & & & $A r$ & $D r$ & $F r$ \\
\hline Lecythis lurida (Miers) S.A.Mori & 23,3962 & 91,05 & 0,506444 & 0,829354 & 0,235979 \\
Tachigali myrmecophila (Ducke) Ducke & 11,2479 & 92,50 & 0,685331 & 0,552728 & 0,474144 \\
Manilkara elata (Allemão ex Miq.) Monach. & 26,3379 & 94,82 & 0,610475 & 0,694997 & 0,379867 \\
Não Identificada1 & 10,6998 & 92,78 & 0,557279 & 0,674294 & 0,484528 \\
Piptadenia suaveolens Miq. & 8,64411 & 90,27 & 0,467266 & 0,769556 & 0,435253 \\
Virola michelii Heckel & 4,12088 & 88,13 & 0,660701 & 0,508595 & 0,552092 \\
Erisma uncinatum Varm. & 96,7648 & 98,09 & 0,505260 & 0,818344 & 0,273908 \\
Carapa guianensis Aubl. & 54,8722 & 98,51 & 0,686311 & 0,584263 & 0,433145 \\
Bertholletia excelsa Bonpl. & 14,7874 & 97,68 & 0,253424 & 0,923704 & 0,287312 \\
Tachigali chrysophylla (Poepp.) Zarucchi \& & 13,9974 & 94,75 & 0,711478 & 0,501499 & 0,492239 \\
Herend. & & & & &
\end{tabular}

Em que: $A r=$ Abundância relativa, $D r=$ Dominância relativa e $F r=$ Frequência relativa.

As análises de componentes principais para as 157 espécies mostraram que apenas Swartzia arborescens (Aubl.) Pittier, Pachira aquatica Aubl., Tachigali alba Ducke, Glycydendron amazonicum Dicke, Bowdichia nitida Spruce ex Berth., Mezilaurus itauba (Meisn.) Taub. ex Mez, Cedrela odorata L. e Virola michelii Heckel tiveram o $1^{\circ}$ componente principal explicando menos de $90 \%$ da variância total, ou seja, apenas 8 em um universo de 157 espécies, o que demonstra que o primeiro componente principal pode ser considerado um excelente Índice de Valor de Importância.

Para ilustrar a metodologia aplicada neste trabalho, utilizou-se a espécie Lecythis lurida, devido a sua ocorrência em todos os 36 conglomerados.

Na matriz de correlações da espécie Lecythis lurida (Tabela 2), verifica-se que não ocorreram valores menores que 0,30 . A aplicação do teste $\mathrm{F}$ de Snedecor mostrou que todas as correlações são diferentes de zero (p-valor $<0,00001$ ), demonstrando serem significativas as inter-relações entre as variáveis $A_{r}, F_{r}$ e $D_{r}$. $\mathrm{O}$ teste de esfericidade de Bartlett apresentou um valor calculado igual a 83,4322 (p-valor $<0,00001$ ), o 
qual permite concluir pela rejeição da hipótese de que a matriz de correlações corresponde a uma identidade. Nesse caso, podem-se aplicar as técnicas de análise de componentes principais e de fatores, pois as variáveis originais são consideradas dependentes.

TABELA 2: Matrizes de covariâncias e de correlações de Lecythis lurida.

TABLE 2: Covariance and correlation matrices of Lecythis lurida.

\begin{tabular}{|c|c|c|c|c|c|c|}
\hline \multirow{2}{*}{ Coeficientes } & \multicolumn{3}{|c|}{ Matriz de covariâncias } & \multicolumn{3}{|c|}{ Matriz de correlações } \\
\hline & $A r$ & $D r$ & $F r$ & $A r$ & $D r$ & $F r$ \\
\hline$A r$ & 7,07783968 & 9,06188111 & 3,17323159 & 1,00000 & $\begin{array}{c}0,83409 \\
<0,0001\end{array}$ & $\begin{array}{l}0,85633 \\
<0,0001\end{array}$ \\
\hline$D r$ & 9,06188111 & 16,6768161 & 4,16725659 & $0.83409<0,0001$ & 1,00000 & $\begin{array}{l}0,73263 \\
<0,0001\end{array}$ \\
\hline$F r$ & 3,17323159 & 4,16725659 & 1,94008849 & $0,85633<0,0001$ & $\begin{array}{l}0,73263 \\
<0,0001\end{array}$ & 1,00000 \\
\hline
\end{tabular}

Em que: $A r=$ Abundância relativa; $D r=$ Dominância relativa e $F r=$ Frequência relativa.

Os valores do coeficiente de confiabilidade de Cronbach foram iguais a 0,841155 para os dados da matriz de covariâncias e de 0,926466 considerando a matriz de correlações, demonstrando que as variáveis apresentam consistência interna ou confiabilidade com relação às escalas utilizadas para medição dos dados.

$\mathrm{O}$ valor da estatística de Kaiser-Meyer-Olkin (KMO) dada pela fórmula 1.3 apresentou um valor igual a 0,71678183 , o que indica a existência de correlações entre as variáveis, recomendando, então, a aplicação da análise de fatores, pois KMO apresentou valor superior a 0,60.

O teste que mede o grau de correlação parcial entre as variáveis (Measure of Sampling AdequacyMSA) apresentou os seguintes resultados: 0,64908; 0,77881050 e 0,74669263, respectivamente para as variáveis $A r, D r$ e $F r$. Como os valores das MSA foram superiores a 0,6, então deve-se usar todas as variáveis $(A r, D r$ e $F r)$ nas análises, ou seja, não é necessário eliminar nenhuma delas.

A Tabela 3 mostra os valores das cargas ou pesos fatoriais, comunalidades e os coeficientes dos escores fatoriais obtidos pela análise de fatores pelo método da máxima verossimilhança a partir da matriz de correlações. Verificou-se, a partir do critério baseado no percentual de variância explicada, a necessidade de apenas um fator, que apresentou uma comunalidade igual a 2,44068178, correspondendo a uma explicação de $81,36 \%$ da variação total. Ressalta-se que, nesta pesquisa, não foi possível aplicar o teste da razão da máxima verossimilhança, segundo Bartlett (1954), expressão 1.6, para conhecer qual o número de fatores $m$ está adequado para reproduzir o modelo, haja vista que o valor do número graus de liberdade de $\chi_{(\mathrm{v}, \alpha)}^{2}$, dado por $v=1 \mid 2\left[(p-m)^{2}-p-m\right]$, ser menor ou igual a zero para $m$. Então, a expressão 1.8 pode ser considerada como uma boa equação para construir índices.

$$
F_{1}=0,86719 \mathrm{Ar}+0,06497 \mathrm{Dr}+0,07708 \mathrm{Fr}
$$

TABELA 3: Valores das cargas fatoriais, comunalidades e dos coeficientes fatoriais.

TABLE 3: Values of factor loadings, communalities and factor coefficients.

\begin{tabular}{cccc}
\hline Variáveis & Cargas fatoriais & Comunalidades & Coeficientes dos escores \\
\hline$A r$ & 0,98738 & 0,97492042 & 0,86719 \\
$D r$ & 0,84475 & 0,71359811 & 0,06497 \\
$F r$ & 0,86727 & 0,75216325 & 0,07708 \\
\hline Total & & 2,44068178 & \\
\hline
\end{tabular}

Em que: $A r=$ Abundância relativa; $D r=$ Dominância relativa e $F r=$ Frequência relativa.

A espécie Lecythis lurida apresentou os seguintes resultados para os três componentes principais:

$$
C P_{1}=0,506444 A r+0,829354 D r+0,235979 F r \text { (autovalor igual a } 23,3962 \text { ) }
$$




$$
\begin{gathered}
C P_{2}=0,724313 A r-0,557663 D r+0,405441 F r(\text { autovalor igual a } 1,8772) \\
C P_{3}=-0,467851 A r+0,034410 D r+0,883137 F r(\text { autovalor igual a } 0,4214)
\end{gathered}
$$

O primeiro componente principal $\left(C P_{1}\right)$ apresentou um autovalor igual a 23,3962 que correspondeu a uma contribuição de $91,05 \%$ para a variância total. $O$ valor do autovalor para o segundo componente principal $\left(\mathrm{CP}_{2}\right)$ foi de 1,8772 , com participação de $7,31 \%$, enquanto o autovalor do terceiro componente principal $\left(\mathrm{CP}_{3}\right)$ foi de 0,4214 que consistiu em uma contribuição de apenas $1,64 \%$. Os valores de $C P_{1} \mathrm{e}$ $C P_{2}$ explicaram conjuntamente $98,36 \%$ da dispersão total. Os valores dos índices de valor de importância baseados em $C P_{1}=0,506444 \mathrm{Ar}+0,829354 \mathrm{Dr}+0,235979 \mathrm{Fr}$, considerando os 36 conglomerados, estão apresentados na Tabela 4. Na coluna CCP1 está a classificação hierárquica em função da magnitude dos resultados. Portanto, o primeiro componente principal pode ser considerado como uma boa equação para expressar o índice de valor de importância, pois explicou 91,05 \% da dispersão total.

Por outro lado, optou-se pela obtenção do índice dado pela expressão 1.7 e definido como o módulo da resultante da soma vetorial dos escores dos componentes principais, ponderados pela contribuição em porcentagem dos respectivos componentes principais à variância total. Os valores deste índice (CPsv) para os 36 conglomerados são mostrados na Tabela 4. Na coluna CCPsv está a classificação hierárquica em função da magnitude dos resultados.

$$
C P s v=\sqrt{0,9105 C P_{1}^{2}+0,0731 C P_{2}^{2}+0,0164 C P_{3}^{2}}
$$

Para mostrar as metodologias descritas e efetivar uma comparação entre os índices $I V I$, escore do $C P 1$, a soma vetorial considerando os escores dos três componentes principais $(C P 1, C P 2, C P 3)$ e o escore $F_{l}$, em termos de classificação pela magnitude para os 36 conglomerados, considerou-se a espécie Lecythis lurida. As observações concernentes às variáveis $A r, F r$ e $D r$ estão inseridas na Tabela 4.

As porcentagens de coincidências considerando o índice $I V I=A r+D R+F r \quad$ com relação aos índices $C P 1(36,1 \%)$, CPsv $(36,1)$ e $\mathrm{F}_{1}(25,0 \%)$ (Tabela 5) são inexpressivas para afirmar que podem produzir resultados similares. Os índices $C P 1$ e CPsv podem ser considerados similares, pois apresentaram uma coincidência nas classificações de $88,9 \%$. Este resultado deve-se ao fato do primeiro componente ter contribuído com $91,05 \%$ da variância total, demonstrando que o $C P 1$ é uma boa escolha como índice de valor de importância. Comparando-se $C P 1$ com $F_{l}$ obteve-se apenas 19,4 \% de coincidências na classificação, concluindo-se que, apesar de serem técnicas multivariadas, apresentaram resultados diferentes. Apenas seis conglomerados tiveram a mesma classificação, considerando-se todos os índices, ou seja, uma porcentagem de coincidências de $16,7 \%$.

TABELA 5: Número e porcentagens de classificações coincidentes dos diversos índices.

TABLE 5: Number and percentage of coincident classifications of various rates.

\begin{tabular}{cccc}
\hline & $C P 1$ & $\mathrm{CPsv}$ & $\mathrm{F}_{1}$ \\
\hline$I V I$ & $13(36,1 \%)$ & $13(36,1 \%)$ & $9(25,0 \%)$ \\
$C P 1$ & & $32(88,9 \%)$ & $7(19,4 \%)$ \\
CPsv & & & $7(19,4 \%)$ \\
\hline
\end{tabular}

Em que: $I V I$ é o Índice de Valor de Importância segundo Curtis e McIntosh (1950); CPI é o Índice de Valor de Importância segundo o primeiro componente principal; CPsv é o Índice de Valor de Importância calculado pela expressão 1.7 e $F_{l}$ é o Índice de Valor de Importância segundo o primeiro fator.

Os valores das cargas fatoriais expressam que as correlações simples entre as variáveis originais $A r$, $\mathrm{Dr}$ e $\mathrm{Fr}$ e a latente $F_{l}$ correspondem, respectivamente, a $98,74 \%, 84,5 \%$ e $86,7 \%$. 
TABELA 4: Comparação da importância de Lecythis lurida em termos de IVI (CIVI), escore do CPI (CCP1), soma vetorial CPsv dos escores dos CP1, CP2 e CP3 (CCpv), escore $F_{1}\left(\mathrm{CF}_{1}\right)$.

TABLE 4: Comparison of the importance of Lecythis lurida according to IVI (CIVI), score CP1 (CCP1), vetorial sum CPsv of the scores of CP1,CP2 e CP3 (CCpv), score F1 (CF1).

\begin{tabular}{|c|c|c|c|c|c|c|c|c|c|c|c|}
\hline $\mathrm{C}$ & $A r$ & $D r$ & $F r$ & $I V I$ & CIVI & $C P 1$ & CCP1 & CPsv & CCPsv & $F_{1}$ & $C F_{1}$ \\
\hline 1 & 3,77 & 6,50 & 4,40 & 14,7 & $32^{\circ}$ & 8,3384 & $29^{\circ}$ & 7,9658 & $30^{\circ}$ & 4,0308 & $34^{\circ}$ \\
\hline 2 & 8,62 & 10,70 & 7,98 & 27,3 & $8^{\circ}$ & 15,1227 & $9^{\circ}$ & 14,4678 & $9^{\circ}$ & 8,7855 & $9^{\circ}$ \\
\hline 3 & 7,50 & 6,57 & 5,85 & 19,9 & $21^{\circ}$ & 10,6277 & $19^{\circ}$ & 10,2054 & $19^{\circ}$ & 7,3817 & $16^{\circ}$ \\
\hline 4 & 9,57 & 15,08 & 7,27 & 31,9 & $5^{\circ}$ & 19,0689 & $5^{\circ}$ & 18,2026 & $5^{\circ}$ & 9,8391 & $5^{\circ}$ \\
\hline 5 & 12,3 & 14,57 & 7,60 & 34,5 & $4^{\circ}$ & 20,1165 & $4^{\circ}$ & 19,2247 & $4^{\circ}$ & 12,2162 & $4^{\circ}$ \\
\hline 6 & 8,96 & 7,20 & 5,99 & 22,2 & $14^{\circ}$ & 11,9226 & $14^{\circ}$ & 11,4548 & $14^{\circ}$ & 8,6995 & $10^{\circ}$ \\
\hline 7 & 6,19 & 6,08 & 5,17 & 17,4 & $26^{\circ}$ & 9,3974 & $25^{\circ}$ & 9,0116 & $25^{\circ}$ & 6,1614 & $23^{\circ}$ \\
\hline 8 & 5,59 & 11,73 & 4,96 & 22,3 & $13^{\circ}$ & 13,7298 & $11^{\circ}$ & 13,1046 & $11^{\circ}$ & 5,9920 & $24^{\circ}$ \\
\hline 9 & 2,84 & 2,42 & 3,20 & 8,46 & $36^{\circ}$ & 4,2005 & $36^{\circ}$ & 4,0496 & $36^{\circ}$ & 2,8667 & $36^{\circ}$ \\
\hline 10 & 5,00 & 7,08 & 3,82 & 15,9 & $29^{\circ}$ & 9,3055 & $26^{\circ}$ & 8,8870 & $26^{\circ}$ & 5,0904 & $30^{\circ}$ \\
\hline 11 & 8,02 & 9,42 & 5,41 & 22,9 & $11^{\circ}$ & 13,1508 & $12^{\circ}$ & 12,5717 & $12^{\circ}$ & 7,9839 & $11^{\circ}$ \\
\hline 12 & 3,29 & 4,06 & 3,11 & 10,5 & $35^{\circ}$ & 5,7673 & $34^{\circ}$ & 5,5185 & $34^{\circ}$ & 3,3566 & $35^{\circ}$ \\
\hline 13 & 7,34 & 6,41 & 6,63 & 20,4 & $19^{\circ}$ & 10,5980 & $20^{\circ}$ & 10,1889 & $20^{\circ}$ & 7,2927 & $18^{\circ}$ \\
\hline 14 & 7,66 & 8,11 & 5,38 & 21,2 & $16^{\circ}$ & 11,8750 & $15^{\circ}$ & 11,3658 & $15^{\circ}$ & 7,5843 & $13^{\circ}$ \\
\hline 15 & 4,72 & 4,13 & 4,55 & 13,4 & $33^{\circ}$ & 6,8894 & $33^{\circ}$ & 6,6271 & $33^{\circ}$ & 4,7122 & $32^{\circ}$ \\
\hline 16 & 7,91 & 7,24 & 5,23 & 20,8 & $20^{\circ}$ & 11,2447 & $17^{\circ}$ & 10,7801 & $17^{\circ}$ & 7,7330 & $12^{\circ}$ \\
\hline 17 & 6,92 & 4,88 & 6,30 & 18,1 & $24^{\circ}$ & 9,0385 & $27^{\circ}$ & 8,7293 & $27^{\circ}$ & 6,8036 & $22^{\circ}$ \\
\hline 18 & 5,71 & 5,43 & 3,94 & 15,1 & $31^{\circ}$ & 8,3249 & $30^{\circ}$ & 7,9783 & $29^{\circ}$ & 5,6081 & $28^{\circ}$ \\
\hline 19 & 12,8 & 14,52 & 8,16 & 35,5 & $3^{\circ}$ & 20,4604 & $3^{\circ}$ & 19,5624 & $3^{\circ}$ & 12,6897 & $3^{\circ}$ \\
\hline 20 & 13,1 & 15,40 & 7,57 & 36,1 & $2^{\circ}$ & 21,1928 & $2^{\circ}$ & 20,2512 & $2^{\circ}$ & 12,9442 & $2^{o}$ \\
\hline 21 & 4,64 & 8,05 & 5,29 & 18,0 & $25^{\circ}$ & 10,2745 & $23^{\circ}$ & 9,8143 & $23^{\circ}$ & 4,9545 & $31^{\circ}$ \\
\hline 22 & 5,93 & 5,74 & 4,64 & 16,3 & $28^{\circ}$ & 8,8586 & $28^{\circ}$ & 8,4934 & $28^{\circ}$ & 5,8730 & $25^{\circ}$ \\
\hline 23 & 6,82 & 5,80 & 6,67 & 19,3 & $22^{\circ}$ & 9,8382 & $24^{\circ}$ & 9,4703 & $24^{\circ}$ & 6,8052 & $21^{\circ}$ \\
\hline 24 & 6,98 & 7,59 & 5,99 & 20,6 & $18^{\circ}$ & 11,2433 & $18^{\circ}$ & 10,7683 & $18^{\circ}$ & 7,0078 & $20^{\circ}$ \\
\hline 25 & 7,49 & 7,59 & 6,63 & 21,7 & $15^{\circ}$ & 11,6526 & $16^{\circ}$ & 11,1733 & $16^{\circ}$ & 7,4994 & $15^{\circ}$ \\
\hline 26 & 5,88 & 4,42 & 6,21 & 16,5 & $27^{\circ}$ & 8,1091 & $32^{\circ}$ & 7,8337 & $31^{\circ}$ & 5,8649 & $26^{\circ}$ \\
\hline 27 & 4,67 & 2,39 & 5,20 & 12,3 & $34^{\circ}$ & 5,5743 & $35^{\circ}$ & 5,4459 & $35^{\circ}$ & 4,6059 & $33^{\circ}$ \\
\hline 28 & 9,73 & 12,49 & 6,50 & 28,7 & $7^{\circ}$ & 16,8202 & $7^{\circ}$ & 16,0680 & $7^{\circ}$ & 9,7503 & $6^{\circ}$ \\
\hline 29 & 13,7 & 19,48 & 8,97 & 42,2 & $1^{\circ}$ & 25,2159 & $1^{\mathrm{o}}$ & 24,0738 & $1^{\mathrm{o}}$ & 13,8462 & $1^{\mathrm{o}}$ \\
\hline 30 & 6,96 & 13,08 & 6,29 & 26,3 & $9^{\circ}$ & 15,8571 & $8^{\circ}$ & 15,1352 & $8^{\circ}$ & 7,3703 & $17^{\circ}$ \\
\hline 31 & 7,49 & 8,33 & 6,82 & 22,6 & $12^{\circ}$ & 12,3112 & $13^{\circ}$ & 11,7918 & $13^{\circ}$ & 7,5621 & $14^{\circ}$ \\
\hline 32 & 9,22 & 14,57 & 7,59 & 31,4 & $6^{\circ}$ & 18,5442 & $6^{\circ}$ & 17,7042 & $6^{\circ}$ & 9,5271 & $7^{\circ}$ \\
\hline 33 & 5,50 & 7,50 & 5,65 & 18,7 & $23^{\circ}$ & 10,3389 & $22^{\circ}$ & 9,8875 & $22^{\circ}$ & 5,6923 & $27^{\circ}$ \\
\hline 34 & 5,19 & 5,26 & 4,79 & 15,2 & $30^{\circ}$ & 8,1212 & $31^{\circ}$ & 7,7894 & $32^{\circ}$ & 5,2117 & $29^{\circ}$ \\
\hline 35 & 7,27 & 6,31 & 7,06 & 20,6 & $17^{\circ}$ & 10,5811 & $21^{\circ}$ & 10,1806 & $21^{\circ}$ & 7,2586 & $19^{\circ}$ \\
\hline 36 & 9,25 & 9,22 & 6,67 & 25,1 & $10^{\circ}$ & 13,9052 & $10^{\circ}$ & 13,3205 & $10^{\circ}$ & 9,1347 & $8^{\circ}$ \\
\hline
\end{tabular}

Em que: $\mathrm{C}=$ Conglomerado; $A r=$ Abundância relativa, $D r=$ Dominância relativa, $F r=$ Frequência relativa da espécie Lecythis lurida; IVI é o Índice de Valor de Importância segundo Curtis e McIntosh (1950) e CIVI é sua classificação hierárquica por conglomerado; $C P 1$ é o Índice de Valor de Importância, segundo o primeiro componente principal e CCP1 é a sua classificação hierárquica por conglomerado; CPsv é o Índice de Valor de Importância calculado pela expressão 1.7 e CCPsv é a sua classificação hierárquica por conglomerado; $F_{1}$ é o Índice de Valor de Importância, segundo o primeiro fator e $\mathrm{CF}_{1}$ é a sua classificação hierárquica por conglomerado. 


\section{CONCLUSÕES}

Conclui-se que o planejamento da estrutura amostral por conglomerado com subdivisões das subparcelas propiciou uma estratificação estrutural-florística do povoamento, principalmente caracterizando que espécies consideradas como de menor importância para a estrutura florestal na área como um todo, apresentam grande relevância em determinadas subáreas da população;

As equações lineares obtidas através da análise de componentes principais explicaram mais de 90 $\%$ da variância dos dados originais, podendo-se concluir que o primeiro componente principal é uma boa escolha como um índice de valor de importância para caracterizar a estrutura florestal, pois apresenta a vantagem de absorver a maior parte da variabilidade apresentada pelas variáveis estudadas;

A equação linear correspondente ao primeiro fator obtido pelo método da máxima verossimilhança para a espécie Lecythis lurida é uma boa escolha como um índice de valor de importância, visto que apresentou uma comunalidade igual a 2,44068178, correspondendo a uma explicação de $81,36 \%$ da variação total.

\section{REFERÊNCIAS}

BARTLETT, M. S. A note on multiplying factors for various chi-squared approximations. Jornal of the Royal Statistical Society, London, v. 16, p. 296-298, 1954.

CAIN, S. A. et al. Application of some phytosociological techniques to Brazilian rain forest. American Journal of Botany, New York, v. 43, p. 911-941, 1956.

CORRAR. L. J.; PAULO, E.; DIAS FILHO, J. M. Análise multivariada para os cursos de administração, ciências contábeis e economia. São Paulo: Atlas, 2007. 344 p.

CRONBACH, L. J. Coefficient alpha and the internal structure of tests. Psychometrika, London, v. 16, n. 3, p. 297-334, 1951.

CURTIS, J. T.; MCINTOSH, R. P. The interrelations of certain analytic and synthetic phytosociological characters. Ecology, Washington, v. 31, p. 434-455, 1950.

FÁVERO, L. P. et al. Análise de dados: modelagem para análise de dados. Rio de janeiro: Elsevier, 2009. $646 \mathrm{p}$.

FINOL, U. H. Nuevos parametros a considerarse en el analysis estructural de Las Selvas Virgenes Tropicales. Revista Forestal Venezolana, Mérida, v. 14, p. 29-42, 1971.

HAIR JUNIOR, J. F. et al. Ensaio sobre la Estructura Florística de la Parte Sub-Oriental del Bosque Universitário "El Caimital" Estado Barinas. Revista Forestal Venezolana, Mérida, v. 7, n. 10-11, p. 77-119, 1964.

LAWLEY, D. N. The estimation of factor looding by the method of maximum likelihood. Proceedings of the Royal Society of Edinburgh, Edinburgh, v. 60, p. 64-82, 1940.

MAROCO, J. Análise estatística com utilização do SPSS. 3. ed. Lisboa: Edições Sílabo, 2007. 824 p.

MINGOTI, S. A. Análise de dados através de Métodos de Estatística Multivariada: uma abordagem aplicada. Belo Horizonte: UFMG, 2005. 300 p.

PEREIRA JÚNIOR, R. A. A utilização da fitossociologia como uma ferramenta na seleção de espécies para exploração e definição de tratamentos silviculturais em floresta de terra-firme sob manejo florestal, na Amazônia brasileira. 2004. 115 f. Dissertação (Mestrado em Ciências Florestais) Universidade Federal Rural da Amazônia, Belém, 2004.

QUEIROZ, W. T. Efeitos da variação estrutural em unidades amostrais na aplicação de processos de amostragem em conglomerados nas Florestas do Planalto do Tapajós. 1977. 109 f. Dissertação (Mestrado em Engenharia Florestal) - Universidade Federal do Paraná, Curitiba, 1977.

QUEIROZ, W. T. Análise de fatores ("Factor Analysis") pelo método da máxima verossimilhança: aplicação ao estudo da estrutura de florestas tropicais. 1984. 112 f. Tese (Doutorado em Estatística e Experimentação Agronômica) - Escola Superior de Agricultura "Luiz de Queiroz", Piracicaba, 1984.

QUEIROZ, W. T. Inventário Florestal: processo de pós-estratificação multidimensional. Boletim da Faculdade de Ciências Agrárias do Pará, Belém, v. 23, p. 7-21, 1995.

PROJETO RADAMBRASIL. Folha S/A 21-Santarém. Rio de Janeiro: Departamento Nacional de 
Produção Mineral, 1976. (Levantamento de Recursos Naturais, 10).

SALOMÃO, R. P. et al. Análise fitossociológica de floresta ombrófila densa e determinação de espécieschave para recuperação de área degradada através da adequação do índice de valor de importância. Boletim do Museu Paraense Emílio Goeldi. Ciências Naturais, Belém, v. 7, n. 1, p. 57-102, 2012.

THONSOM, G. H. The factorial analysis of human ability. London: University of London, 1951.

VALENTE, M. D. R. et al. Modelo de predição para o volume total de Quaruba (Volchysia inundata ducke) via análise de fatores e regressão. Revista Árvore, Viçosa, v. 35, n. 2, p. 307-317, 2011. 